Document downloaded from:

http://hdl.handle.net/10251/180904

This paper must be cited as:

Martinez-Roman, J.; Puche-Panadero, R.; Terron-Santiago, C.; Sapena-Bano, A.; BurrielValencia, J.; Pineda-Sanchez, M. (2021). Low-Cost Diagnosis of Rotor Asymmetries of Induction Machines at Very Low Slip With the Goertzel Algorithm Applied to the Rectified Current. IEEE Transactions on Instrumentation and Measurement. 70:1-11.

https://doi.org/10.1109/TIM.2021.3115216

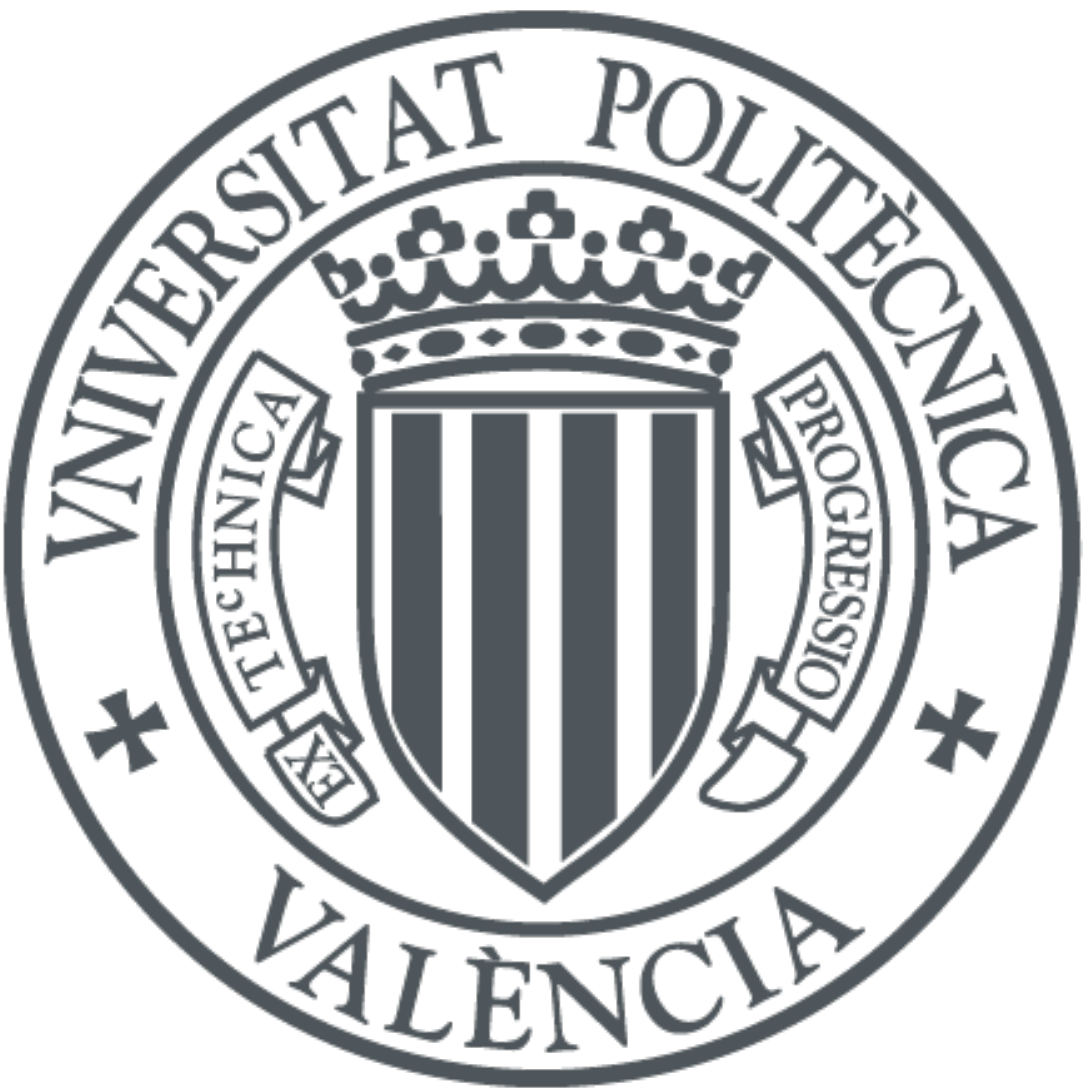

The final publication is available at

https://doi.org/10.1109/TIM.2021.3115216

Copyright Institute of Electrical and Electronics Engineers

Additional Information 


\title{
Low-Cost Diagnosis of Rotor Asymmetries of Induction Machines at Very Low Slip with the Goertzel Algorithm Applied to the Rectified Current
}

\author{
Javier Martinez-Roman, Member, IEEE, Ruben Puche-Panadero, Member, IEEE, Carla Terron-Santiago, Angel \\ Sapena-Bano, Jordi Burriel-Valencia, and Manuel Pineda-Sanchez, Member, IEEE.
}

\begin{abstract}
Induction machines are essential components of many industrial installations and, therefore, their faults must be detected early. Fault detection using current spectrum analysis is attracting an increasing interest as a condition-based monitoring technique. However, its use to detect rotor asymmetries in high-power induction machines, which operate at very low slip, is particularly challenging, due to the closeness of the characteristic fault harmonics to the fundamental component, separated only a few $\mathrm{mHz}$. Their reliable detection in harsh industrial environments requires a very high spectral resolution, that is, long acquisition times and a huge number of current samples, what hinders its implementation on embedded, online devices with limited computing resources. To address this problem, this paper presents a novel combination of diagnostic techniques, the use of the rectified current as diagnostic signal, and the Goertzel algorithm as signal processing tool. This unique combination allows for an optimized implementation ot the Goertzel algorithm, which provides a high spectral resolution in the full load range of the machine, with a low computational cost and a negligible memory footprint. This proposal is validated experimentally with the fault diagnosis of a high-power mediumvoltage industrial motor.
\end{abstract}

Index Terms-Condition monitoring, Goertzel algorithm, induction machines, spectral analysis.

\section{INTRODUCTION}

I NDUCTION machines (IMs) are prevalent in industrial processes, mainly due to their reliability and low maintenance. For that reason, IM faults can cause unanticipated and costly stops of production lines [1]. Therefore, there is a growing interest in the development of condition-based monitoring (CBMs) methods that are able to detect IM faults on line [1], to avoid the sudden stop of IM powered industrial processes [2].

Motor current signature analysis (MCSA) has become a successful technique for diagnosing IM failures, as it is noninvasive and its implementation requires low hardware and software resources [3], [4]. Besides, MCSA can identify the current components corresponding to multiple types of faults, even if they are simultaneous.

This work was supported by the Spanish "Ministerio de Ciencia, Innovación y Universidades (MCIU)", the "Agencia Estatal de Investigación (AEI)" and the "Fondo Europeo de Desarrollo Regional (FEDER)" in the framework of the "Proyectos I+D+i - Retos Investigación 2018", project reference RTI2018102175-B-I00 (MCIU/AEI/FEDER, UE).

The authors are with the Institute for Energy Engineering, Universitat Politècnica de València, 46022 Valencia, Spain (e-mail: jmroman@die.upv.es, rupucpa@die.upv.es, cartersa@alumni.upv.es, asapena@die.upv.es, jorburva@die.upv.es, mpineda@die.upv.es).
One of the most common types of IM faults is rotor asymmetries, that is, rotor bar and end-ring breakages, or asymmetrical resistances of the rotor windings [5]. They can be produced by a mismatch in the phase windings of wounded rotor IMs, such as doubly-fed induction generators (DFIGs), or by poorly manufactured rotor cages of squirrel cage induction motors (SCIMs), which may lead to rotor breakages due to the high mechanical and thermal stresses that the rotor cage supports, specially in the start-up process. Fig. 1 shows the rotor cage of the high-power (3.15 MW) SCIM used for the validation of this work, with a broken bar fault.

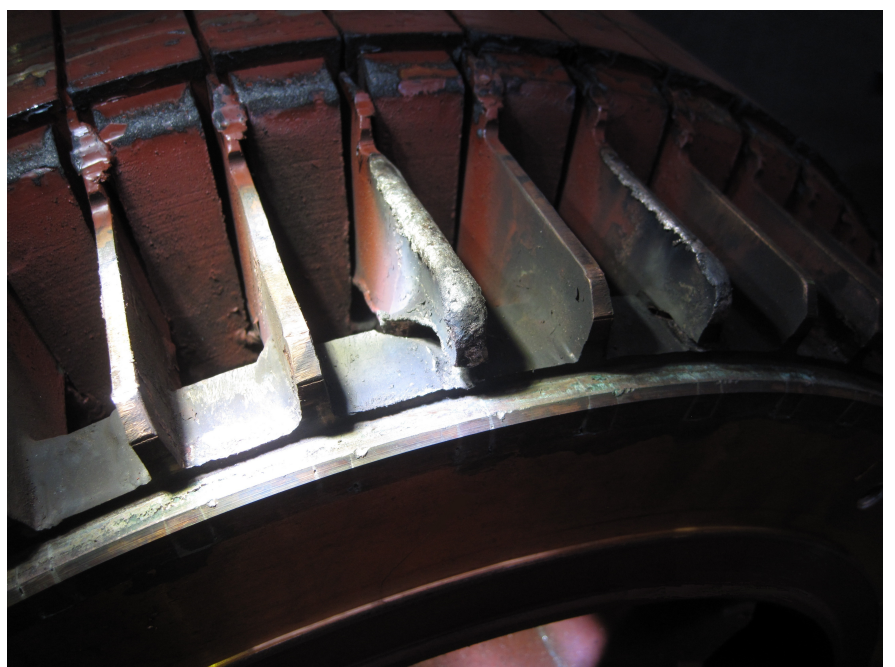

Fig. 1. Motor of Appendix A (3.15 MW), with a broken bar fault

Broken rotor bars, broken end-rings, or asymmetrical resistances of rotor windings, induce in the stator current an amplitude modulation, with a set of characteristic frequencies given by [6], [7]

$$
f_{\text {asym }}=2 k s f_{1}, \quad k=1,2, \ldots,
$$

where $f_{1}$ is the supply frequency, and $s$ is the rotor slip. The stator current of the IM with a rotor asymmetry can be approximated, using (1), as

$$
i(t)=I \cos \left(\omega_{1} t\right) \cdot\left(1+\beta \cos \left(2 \pi f_{\text {asym }} t\right)\right),
$$

where $\omega_{1}=2 \pi f_{1}, I$ is the amplitude of the fundamental current component, and $\beta$ is the amplitude of the fault component, in per-unit of the fundamental one. In case of incipient faults 
this factor can be very small $(\beta \ll 1)$, less than $\beta=1 / 200$ for partially broken cage bars [2].

The amplitude modulation given by (2) results in sideband fault components symmetrically located around the fundamental component, at frequencies

$$
f_{b b}=f_{1} \pm f_{\text {asym }}=f_{1} \pm 2 k s f_{1} \quad k=1,2, \ldots
$$

If only the main fault components are considered, those with harmonic order $k=1$ in (1), these sideband components appear at a distance $2 s f_{1}$ from the fundamental, as

$$
i(t)=I\left(\cos \left(\omega_{1} t\right)+\frac{\beta}{2} \cos \left(\omega_{1}(1 \pm 2 s) t\right)\right) .
$$

The direct use of (4) for the diagnosis of rotor asymmetries is especially challenging for large IMs [8], inverter fed IMs [9], or small IMs with light loads [8], [10]. In these cases, the rotor slip $s$ can be extremely low and, therefore, the distance $2 s f_{1}$ can be very small. For example, the $3.15 \mathrm{MW}$ IM tested in this work (see Appendix A) has a rated slip of just $s_{n}=0.006$, and a distance of just $0.6 \mathrm{~Hz}$ between the fundamental and the fault components at rated load. A so tiny spectral distance poses severe difficulties for the successful application of MCSA [10]:

- To achieve such a high spectral resolution $(\Delta f)$, in the range of hundredths of a hertz, long sampling times are required [11], because it is the inverse of the acquisition time $T_{a c q}$, that is, $\Delta f=1 / T_{a c q}$. Another requirement for the practical implementation of MCSA is to avoid aliasing artifacts, which can make the fault harmonics appear at non-realistic frequencies. This problem can be solved with analog anti-aliasing filters [12]. Nevertheless, as modern low cost analog-to-digital converters (ADCs) can operate at very high speed, it is common to sample the current with a very high frequency, followed by a digital low pass filter. The combination of a high sample rate and long acquisition times produces a large number of current samples. For the motor used in this work (see Appendix A), an acquisition time of $T_{a c q}=100 \mathrm{~s}$ is needed to obtain a resolution of $0.01 \mathrm{~Hz}$. As a sampling frequency $f_{s}=5000 \mathrm{~Hz}$ has been used, this gives a total number of $N_{s}=T_{a c q} \cdot f_{s}=100 \cdot 5000=500000$ samples, which must be stored and processed with the fast Fourier transform (FFT). In case of low cost, embedded devices, such as field programmable gate arrays (FPGAs) or digital signal processors (DSPs), this considerable computing burden and memory footprint hampers its use for the fault diagnosis of high power induction machines [7], [13]. Moreover, a growing trend is to add fault diagnostic capabilities to variable speed drive (VSD) controllers or to programmable logic controllers (PLCs) [14], what requires using the least possible resources of the controller to avoid disturbing its normal operation. Besides, diagnostic data must be sent to servers, which makes it necessary to reduce the volume of data transmitted for lower communication costs.

- This high spectral resolution is only needed in a very narrow spectral band, just $1.2 \mathrm{~Hz}$ in the case of the experimental motor of Appendix A. Therefore, from a diagnostic perspective, practically the whole spectrum obtained with the FFT is discarded, because it covers the range $\left[0-f_{s} / 2\right]([0-2500 \mathrm{~Hz}]$ in the experimental case).

- The position of this narrow diagnostic band is not constant but is centred on the fundamental frequency.

- The leakage produced by the fundamental component can hide the fault harmonics at so a small distance, making them undetectable, and giving a misdiagnosis.

This challenge is particularly important in case of large, medium voltage (MV) induction motors, such as the one considered in this paper, whose unexpected breakdown can cause extraordinary economic losses, well beyond their high repair expenses [15]. As these motors have very high starting currents, they are started usually with a reduced voltage, with a limited torque. This results in long starting times, with a reduced cooling ability and high bar currents, which produces high thermal and electrical stresses in the rotor cage. As these motors typically have more rotor bars than small machines, an added difficulty is that the flux imbalance generated by a single bar breakage, and therefore its signal-to-noise ratio, is much lower, making it difficult to detect the fault [16]. Besides, as these MV large-sized motors have lower per-unit stator and rotor resistances and a higher rated efficiency than low-voltage small motors [15], their rated slip is very low. These characteristics make the application of MCSA to MV induction motors a challenging task.

Therefore, the problem to solve is how to obtain the fault harmonics generated by rotor asymmetry IM faults with a high spectral resolution, but with a reduced computational cost, and a minimal memory and code footprint. This problem has been addressed using diverse methods in the recent literature. As the FFT is the main signal processing technique used for obtaining the spectral content of the IM current, different works are aimed towards reducing its computational burden. The zoomFFT (ZFFT) [7], [17] can increase the accuracy within a given frequency range, with a reduced sampling frequency. The ZFFT consists in a frequency translation of the current signal, using a digital complex mixer, followed by a decimation stage with an anti-aliasing low-pass filter, which reduces the required number of samples. This reduces the computational cost of the final FFT stage [7] while keeping the same time acquisition period and frequency resolution. The implementation of ultralong FFT using dedicated hardware has also been considered in [18], [19]. Other techniques propose the computation of the Fourier transform (FT) of the current in real-time, as in [20], where the variation of the leakage effect produced by a broken rotor bar is measured in a small time window, using the sliding window discrete Fourier transform (SDFT).

A different line or work is to avoid the use of the FFT. It has been done using multiple signal classification (MUSIC) [21] methods, or estimating signal parameters via rotational invariance technique (ESPRIT) [22]. Other authors propose instead performing the fault diagnosis of IMs in the time domain, using an histogram of oriented gradients in [23], or the analysis of quaternions in [24]. In [15], a combination of frequency and time domain features, extracted from a Hall sensor installed between two stator coils, has been used a an input for intelligent classifiers, able to detect a broken bar fault at very low slip with a short acquisition time. 
A common problem of the aforementioned techniques is the difficulty of implementing them in low-cost processors, due to their complexity of their code or hardware, or due to their computing time or memory burden. To solve these limitations, new techniques with a minimal footprint, in terms of code simplicity, memory usage, and latency, are needed.

In fact, there is an alternative approach to apply MCSA to induction machines at very low slip without using the FFT, followed in this paper. It is based on the Goertzel algorithm [25], which is able to compute directly a set of spectral components of the machine current. If this set is very small, its computational cost is much lower than the FFT of the whole current signal. The Goertzel algorithm has been used for obtaining only the fault harmonics of a permanent magnet synchronous generator in [26], using a bank of filters with a sampling frequency of $1 \mathrm{MHz}$, of an IM with bearing faults in [27] and with broken bars in [28]. Nevertheless, in these works, the exact position of the fault harmonics must be known before applying the Goertzel algorithm, which may result in misdiagnosis in case of small frequency deviations. Besides, in case of a broken bar fault, the current signal must be windowed to avoid the fundamental leakage, which requires storing the whole current signal, as in [28], [29]. These problems are addressed in [30], which avoids the storage of the current signal by windowing it in the frequency domain, and extends the calculation of the spectral components to narrow disjoint frequency bands, to avoid missing the fault harmonics. Nevertheless, the positions and widths of these bands vary with the load of the motor, which makes its application difficult. Besides, in [30] complex number arithmetic is used, which results in high computing and storage costs.

This paper addresses these problems, proposing a unique combination of two diagnostic techniques. On the one hand, the use of the rectified current signal, as in [31], which allows to define a single diagnostic band that covers the whole load range, to halve its width, and to translate its lower frequency to zero frequency. On the other hand, the use of the Goertzel algorithm, implemented using real number arithmetic as in [26], but applied only to the frequency bins of the diagnostic band with minimum leakage, what eliminates the need of storing and windowing the full current signal. The combined use of the rectified current signal and the optimized implementation of the Goertzel algorithm produces a high resolution current spectrum in the full load range of the IM, with a negligible memory and code footprint and a reduced computational burden, what enables its implementation in online low-cost embedded devices, such as FPGAs and DSPs, or in modern smart sensors, mounted on the machine framework.

This paper is structured in the following way. Section II presents the use of the rectified current signal for shifting the signature of the asymmetry fault to a low frequency spectral band and for halving its bandwidth. Section III presents the optimization of the Goertzel algorithm to generate a high resolution spectrum of this band with a low computational burden. In Section IV the proposed technique is experimentally validated with the diagnosis of a broken bar fault in a high power industrial IM. Finally, Section V presents the conclusions.

\section{NARRow-BAND Frequency Analysis of the RECTIFIED CURRENT SIGNAL}

The first step of the proposed approach consists in fixing the position of a narrow diagnostic frequency band that covers the whole load range, and halving its width. This solves the problem of missing the fault harmonics in case of frequency deviations, when their frequencies are the only ones computed, as in [26]-[28], and also eliminates the need of analysing disjoint narrow frequency bands that may contain the fault harmonics, whose position and width is load dependent, as in [30]. This step is done by rectifying the current signal before spectral analysis, either by using a hardware rectifier or by changing the sign of the negative current samples, as in [31].

As mentioned in Section I, the main fault harmonics in (4) can be very close to the fundamental frequency for low slip values, just $2 s f_{1}$ apart. Therefore, for an IM with a low rated slip $s_{n}$, the frequency band of diagnostic interest, where the fault harmonics are expected to show up, has a very small width. Assuming that large induction motors operate at frequencies equal or lower than their rated one, $f_{n}$, and taking into account (1), the width of this diagnostic frequency band can be established as $F_{S}=4 s_{n} f_{n}$, with $F_{S} \ll f_{n} \ll f_{s}$. Therefore, only a narrow-band spectral analysis is needed. Unfortunately, the spectrum obtained with the FFT covers the full spectral range $\left[0-f_{s} / 2\right] \mathrm{Hz}$, thus wasting most of the calculated frequency bins. An additional problem is that this narrow band is centred around the fundamental frequency $f_{1}$, thus its position depends on the value of $f_{1}$. To solve these problems, the band where the fault harmonics are expected to show up is first translated to the origin, and, at the same time, its width is halved, $F_{S}=2 s_{n} f_{n}$, using the rectified current signal, as in [31].

The expression of the rectified current signal in a faulty IM can be obtained as the product of the current signal (2) and its sign, as

$$
|i(t)|=i(t) \cdot \operatorname{sgn}(i(t))=i(t) \cdot \operatorname{sgn}\left(\cos \left(\omega_{1} t\right)\right),
$$

where $\operatorname{sgn}(\cdot)$ stands for the sign function [31]

$$
\operatorname{sgn}(x)=\left\{\begin{array}{cc}
1, & \text { if } x>0 \\
0, & \text { if } x=0 \\
-1, & \text { if } x<0
\end{array}\right.
$$

The square signal given by the expression $\operatorname{sgn}\left(\cos \left(\omega_{1} t\right)\right)$ in (5) can be expressed as

$$
\operatorname{sgn}\left(\cos \left(\omega_{1} t\right)\right)=\frac{4}{\pi} \sum_{h=1,3,5, . .}^{\infty}\left[\frac{\sin \left(h \frac{\pi}{2}\right)}{h} \cos \left(h \omega_{1} t\right)\right] .
$$

and, substituing (7) in (5) gives

$$
\begin{aligned}
|i(t)|= & \frac{4 I}{\pi}\left(\frac{1}{2}+\frac{\beta}{2} \cos \left(2 \pi\left(2 s f_{1}\right) t\right)+\right. \\
& +\sum_{h=3,5, . .}^{\infty}\left[\frac{\sin \left(h \frac{\pi}{2}\right)}{h} \cos \left(h \omega_{1} t\right) \times\left(\cos \left(\omega_{1} t\right)+\right.\right. \\
& \left.\left.\left.+\frac{\beta}{2} \cos \left(\omega_{1}(1 \pm 2 s) t\right)\right)\right]\right) .
\end{aligned}
$$

In the expression of the rectified current signal (8) three terms appear: a direct current (DC) term, $\frac{2 I}{\pi}$, a low frequency component corresponding to the main fault harmonic 
$\frac{2 \beta I}{\pi} \cos \left(2 \pi\left(2 s f_{1}\right) t\right)$, and a set of high frequency harmonic components, centred around $f_{1}, 2 f_{1}, 3 f_{1}, \ldots$ This structure of the rectified current signal of a faulty IM has an important benefit from a diagnostic perspective: the signature of the rotor asymmetry fault in (8) is a single fault component, at a low frequency of $2 s f_{1}$, instead of the two fault harmonics at frequencies $f_{1} \pm 2 s f_{1}$ in (4). Therefore, for a motor with a rated slip $s_{n}$, the frequency band of diagnostic interest is [0 $\left.-2 s_{n} f_{n}\right]$, instead of the frequency band $\left[f_{n}\left(1-2 s_{n}\right)\right.$ $\left.f_{n}\left(1+2 s_{n}\right)\right]$ that must be scanned when analysing directly the non-rectified current signal. Besides, this band starts always at the origin, independently of the fundamental frequency.

The rectification process generates an additional $\frac{4}{\pi}$ factor in (8), compared with (4). Nevertheless, the amplitude of the fault harmonic at frequency $2 s f_{1}$ in (8) is expressed in $\mathrm{dB}$ as

$$
\left.\widehat{i}\left(2 s f_{1}\right)\right|_{\mathrm{dB}}=10 \log \frac{\left|\widehat{i}\left(2 s f_{1}\right)\right|^{2}}{|\widehat{i}(0)|^{2}}=20 \log \frac{\beta}{2},
$$

where $\widehat{i}(f)$ stands for the FT of $|i(t)|$. This eliminates the influence of the additional $\frac{4}{\pi}$ factor.

\section{OPTIMIZED IMPLEMENTATION OF THE GOERTZEL Algorithm ApPlied TO THE RECTIFIEd CURRENT SIGNAL}

Only a narrow-band of frequency width $\left[0-2 s_{n} f_{n}\right]$ of the rectified current is needed to detect the rotor asymmetry fault in an IM with rated slip $s_{n}$. Nevertheless, the FFT computes the frequency bins in the whole range $\left[0-f_{s} / 2\right]$ $\left(f_{s}>>2 s_{n} f_{n}\right)$, so wasting the results obtained outside this diagnostic band. In this work, the Goertzel algorithm is used for computing only the spectrum band of diagnostic interest, and its implementation is optimized to calculate only of the frequency bins with minimum spectral leakage, to avoid the storage of the current signal, and to use only real number arithmetic, which help achieve a negligible memory footprint.

\section{A. The Goertzel Algorithm}

The diagnostic process begins with the acquisition of the current signal. The machine current, $i(t)$, is sampled during an acquisition time $T_{a c q}$ at a rate $f_{s}$, and its absolute value is taken, what gives a time-series of $N=T_{a c q} \cdot f_{s}$ current samples $|i[n]|$, with the time-index $n=0, \ldots, N-1$. The $\mathrm{N}$-point discrete Fourier transform (DFT) of $|i[n]|$ gives a frequencyseries, $\hat{i}[k]$, with the frequency-index $k=0, \ldots, N-1$. Its $k$ th bin is given by

$$
\hat{i}[k]=\sum_{n=0}^{N-1} e^{-j 2 \pi n k / N}|i[n]| .
$$

The FFT is a block algorithm that is able to compute the $N$ points of the DFT (10) in a very effective way. On the contrary, the Goertzel algorithm is able to compute a single output of this N-point DFT. It can be implemented using a second-order infinite impulse response (IIR) filter, as depicted in Fig. 2. Its $z$-domain transfer function is

$$
H_{G}(z)=\frac{1-\left(e^{-j 2 \pi k / N}\right) \cdot z^{-1}}{1-2 \cos (2 \pi k / N) \cdot z^{-1}+z^{-2}}
$$

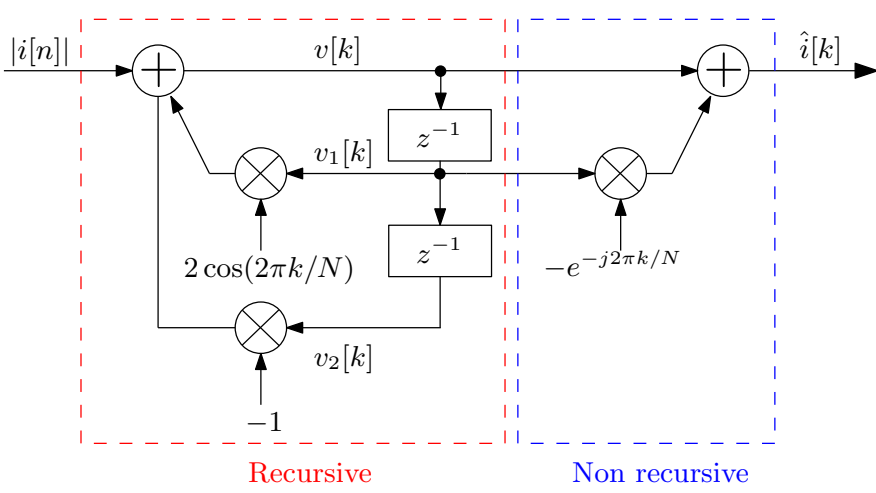

Fig. 2. IIR filter that implements the Goertzel algorithm, applied to the rectified current signal.

The value of $k$ in (11) and in Fig. 2 can be any value between 0 and $N-1$, and is not restricted to integer values [32]. Therefore, (11) gives full flexibility in specifying the frequencies of the bins to be calculated with (11), by adjusting its resonant frequency.

From Fig. 2, it can be seen that the Goertzel algorithm has a recursive part (Fig. 2, left), which must be processed with every current sampled, and thus implemented $N$ times, plus a feed forward part (Fig. 2, right), which needs only be computed after the last current sample has been processed. The present work takes advantage of this structure for proposing the following optimizations, to reduce to a minimum the computing effort and the memory footprint of (11).

\section{B. Optimized Selection of the Frequency Bins Computed with the Goertzel Algorithm}

The frequency bins of the rectified current spectrum with diagnostic interest for an IM with rated slip $s_{n}$, and rated supply frequency $f_{n}$, belong to the frequency band [0 - $\left.2 s_{n} f_{n}\right]$. Within this band, the frequency bins with the lowest leakage from the fundamental component are selected to be processed with (11). The process of sampling the rectified current during a limited acquisition time, $T_{a c q}$, can be modelled as the product of a current signal of infinite duration $\left|i_{\infty}(t)\right|$ and a rectangular time window of length $T_{a c q}$, as

$$
|i(t)|=\left|i_{\infty}(t)\right| \cdot \operatorname{rect}_{T_{a c q}}(t) .
$$

Therefore, the FT of the rectified current can be obtained, using the convolution theorem, as the convolution of the FTs of these two functions,

$$
\mathcal{F}(|i(t)|)=\mathcal{F}\left(\left|i_{\infty}(t)\right|\right) * \mathcal{F}\left(\operatorname{rect}_{T_{a c q}}\right),
$$

where the symbol ' $*$ ' stands for the convolution operation. The FT of a rectangular window is a sinc function. Therefore, as the fundamental component of the current has been displaced to the origin by the rectification process, its leakage at any frequency can be expressed from (13) as the sinc function centred at the origin [8], given by

$$
\mathcal{F}\left(\operatorname{rect}_{T_{a c q}}\right)=\operatorname{sinc}\left(f \cdot T_{a c q}\right)=\frac{\sin \left(\pi f \cdot T_{a c q}\right)}{\pi f \cdot T_{a c q}} .
$$


The leakage generated by the fundamental component is null at the zeros of the sinc function (14), given by the expression

$$
\pi f \cdot T_{a c q}=k \cdot \pi \Longrightarrow f=\frac{k}{T_{a c q}}=k \cdot \Delta f \quad k=0,1, \ldots,
$$

where $\Delta f=1 / T_{a c q}=f_{s} / N$ is the frequency resolution achieved with an acquisition time $T_{a c q}$. Nevertheless, as the width of the frequency band of diagnostic interest is limited to $2 s_{n} f_{n}$, the maximum value of $k$ needed in (15), $k_{\max }$, is given by the condition $k_{\max } \cdot \Delta f \geq 2 s_{n} f_{n}$, that is

$$
k_{\text {max }}=\operatorname{ceil}\left(\frac{2 s_{n} f_{n}}{\Delta f}\right)=\operatorname{ceil}\left(2 s_{n} f_{n} T_{a c q}\right),
$$

where $\operatorname{ceil}(x)$ is the ceiling function, which maps $x$ to the least integer greater than or equal to $x$.

Combining (15) and (16), the Goertzel algorithm is computed only at the frequency bins within the diagnostic band with minimum leakage from the fundamental (see Fig. 3), located at frequencies

$$
f=k \cdot \Delta f \quad k=0,1, \ldots, k_{\max } .
$$

For example, for the motor considered in Appendix A

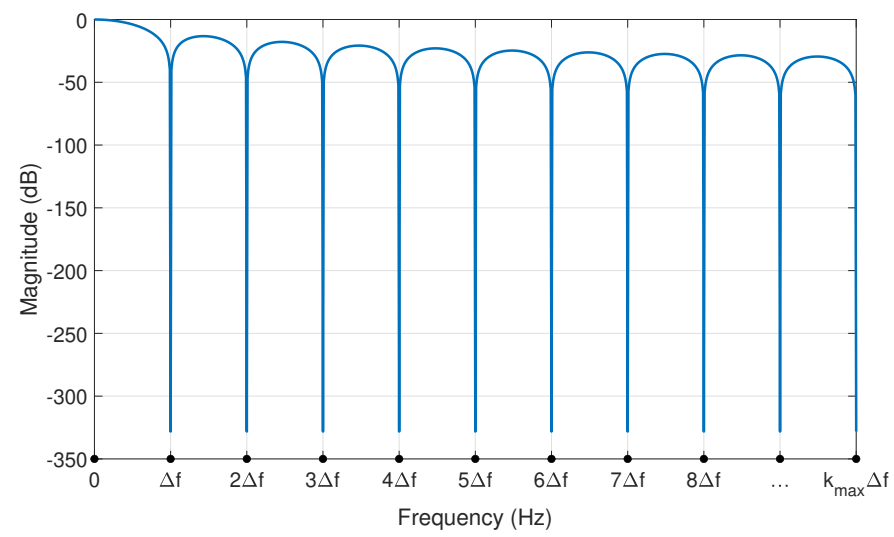

Fig. 3. Spectral leakage generated by the fundamental component of the rectified current signal, located at the origin, and frequency bins selected for calculating the Goertzel algorithm (black dots in the frequency axis), the ones with the lowest leakage. In this plot, $\Delta f=1 / T_{a c q}$ and $k_{\max }=$ $\operatorname{ceil}\left(2 s_{n} f_{n} / \Delta f\right)(16)$.

$\left(s_{n}=0.006, f_{n}=50 \mathrm{~Hz}\right)$, and with an acquisition time $T_{a c q}=100 \mathrm{~s}$, the maximum value of $k$ would be, using (16), $k_{\max }=60$. Therefore, just 61 frequency bins are needed to be processed and stored for diagnostic purposes, much less than the 500000 frequency bins generated by the FFT of the current signal (with a sampling rate $f_{s}=5000 \mathrm{~Hz}$ ).

It is worth mentioning that obtaining the exact position of the frequency bins with a minimum spectral leakage, as given by (17), has been possible thanks to the rectification process, which translates the position of the fundamental component to zero-frequency. The exact location of the leakage-free frequency bins is, in general, not possible for the original, non-rectified current signal, due to the uncertainty on the exact value of its fundamental frequency, where a small error (in the range of the $\mathrm{mHz}$ ) can cause a huge increase of the spectral leakage [8].

\section{Optimized Implementation of the Recursive Part of the Goertzel Algorithm}

The recursive part of the Goertzel algorithm (Fig. 2, left) must be computed for each of the rectified current samples $\left|i_{n}\right|$, with $n=0, \ldots, N-1$, and for each of the desired output frequency bins $\hat{i}[k]$, with $k=0, \ldots, k_{\max }(17)$. This part of the Goertzel algorithm be formulated in a recursive way as

$$
\left\{\begin{aligned}
v_{2}[k] & =v_{1}[k] \\
v_{1}[k] & =v[k] \\
v[k] & =v_{1}[k] \cdot 2 \cos (2 \pi k / N)-v_{2}[k]+\left|i_{n}\right|
\end{aligned}\right.
$$

where $v[k]$ is a buffer of intermediate results, and $v_{1}[k]$ and $v_{2}[k]$ are memory buffers used to store the content of $v[k]$ in the previous recursions, as depicted in Fig. 2.

To optimize the memory footprint, (18) is applied as soon as a new current sample $i_{n}$ is available from the signal acquisition system, and after computing (18) this current sample is discarded. This is possible because the current signal must not be windowed to eliminate the fundamental leakage, thanks to the choice of frequency bins made in Section III-B, and eliminates the need for storing the $N$ samples of the current signal $\left(N=5 \cdot 10^{5}\right.$ in the case of the motor tested in this work). Nevertheless, this approach limits the time available for computing (18) to the time interval between consecutive current samples $(\Delta t=0.2 \mathrm{~ms}$ with the $5 \mathrm{kHz}$ sampling rate used in the experimental tests). To reduce the computation time of (18), the factors $2 \cos (2 \pi k / N)$ are pre-calculated and stored in a memory buffer, what reduces the number of operations per current sample to a single real multiply and two real adds. In this way, the total memory storage needed to implement the optimized recursive part of the Goertzel algorithm, including the twiddle factors $2 \cos (2 \pi k / N)$, is $4\left(k_{\max }+1\right)$ floating point numbers, and the total number of operations is $\left(k_{\max }+1\right)$ real multiplies and $2\left(k_{\max }+1\right)$ real adds per current sample $\left(k_{\max }=60\right.$ in the case of the motor tested in this work).

\section{Optimized Implementation of the Feed Forward Part of the Goertzel Algorithm}

The feed forward part of the Goertzel algorithm (Fig. 2, right) gives the final, complex value of the $k$ th output bin, after iteration $N$, as

$$
\hat{i}[k]=v[k]-e^{j 2 \pi k / N} \cdot v_{1}[k] .
$$

Nevertheless, for diagnostic purposes, only the modulus squared of the Goertzel algorithm output is needed, according to (9), which can be calculated from (19) as [26]

$$
\begin{aligned}
|\hat{i}[k]|^{2} & =\left(v[k]-v_{1}[k] \cos (2 \pi k / N)\right)^{2}+\left(v_{1}[k] \sin (2 \pi k / N)\right)^{2}= \\
& =v[k]^{2}+v_{1}[k]^{2}-2 \cos (2 \pi k / N) \cdot v[k] \cdot v_{1}[k]
\end{aligned}
$$

Using (20), the diagnostic output, in $\mathrm{dB}$ scale, is given by

$$
|\hat{i}[k]|_{\mathrm{dB}}=10 \log \frac{|\hat{i}[k]|^{2}}{|\hat{i}[0]|^{2}} .
$$

Optimizing the calculation of the feed forward part of the Goertzel algorithm for diagnostic purposes as (20) has two advantages: 
- The result of (20) is a real number, not a complex one. Therefore, its computation can be performed in place, using for storing the Goertzel algorithm output the same memory buffer as for $v[k]$, whose values are discarded after the computation. That is, without increasing the memory footprint optimized for the recursive part of the Goertzel algorithm.

- The multiplication by the twiddle factors $2 \cos (2 \pi k / N)$ in (20) can reuse these factors, stored in the pre-calculated buffer used in the recursive part of the Goertzel algorithm, thus reducing the computational cost of (20) to 4 real multiplications and 3 real additions per output bin.

For comparative purposes, the first-order Goertzel algorithm proposed in [30] for fault diagnosis of IMs uses complex arithmetic, requiring four real multiplications and two real additions per current sample and per output bin, rather than one real multiplication and two real additions as in the proposed approach. Besides, all the memory buffers must store complex numbers, what duplicates the memory footprint of the method proposed in this work. Finally, it is applied to the current signal, not the rectified one, so it requires spectral filters to avoid the spectral leakage of the supply component.

\section{E. Flowchart of the Optimized Diagnostic Goertzel algorithm}

Combining (16), (18), (20) and (21), the flow chart of the proposed method is depicted in Fig. 4.

\section{EXPERIMENTAL VALIDATION}

To validate experimentally the proposed method, a large squirrel cage motor ( $6 \mathrm{kV}, 3.15 \mathrm{MW}$, grid connected), whose characteristics are given in Appendix $\mathrm{A}$, has been tested, directly fed from a $50 \mathrm{~Hz}$ power line. It drives a low pressure pump and a high pressure pump in a thermal power plantheating plant (TPP-HP), as seen in Fig. 5. This motor had a broken bar, detected by the proposed approach, and confirmed with the rotor dismounted, as seen in Fig. 6.

The stator current is shown in Fig. 7. It was acquired at a rate of $5 \mathrm{kHz}$, during an acquisition time of $T_{a c q}=100 \mathrm{~s}$, giving $N=5 \cdot 10^{5}$ current samples. During this test, the motor was partially loaded, running at a constant speed of 2994.6 $\mathrm{rpm}(s=0.0018)$, and the measured supply frequency was $f_{1}=49.98 \mathrm{~Hz}$. Applying (1) and (4), the expected frequencies of the three first fault components are given in Table I. Additionally, the current signal depicted in Fig. 7 contains other components generated by electromagnetic noise, and by the non-ideal character of the machine, as seen in the enlarged view of Fig. 7. The proposed method is validated in the presence of these additional harmonic components, unavoidable in industrial machines.

The conventional spectrum of the current, shown in Fig. 8, has been obtained using a Hanning window. The theoretical broken bar fault harmonics calculated in Table I, second row, around the supply frequency, appear clearly in this spectrum, what indicates a the broken bar fault, confirmed by visual inspection. Nevertheless, the memory requirement for this FFT of $5 \cdot 10^{5}$ samples is, at least, $3 \cdot 5 \cdot 10^{5}$ double precision floating point numbers [33], that is, $11.72 \mathrm{MB}$ of memory storage.

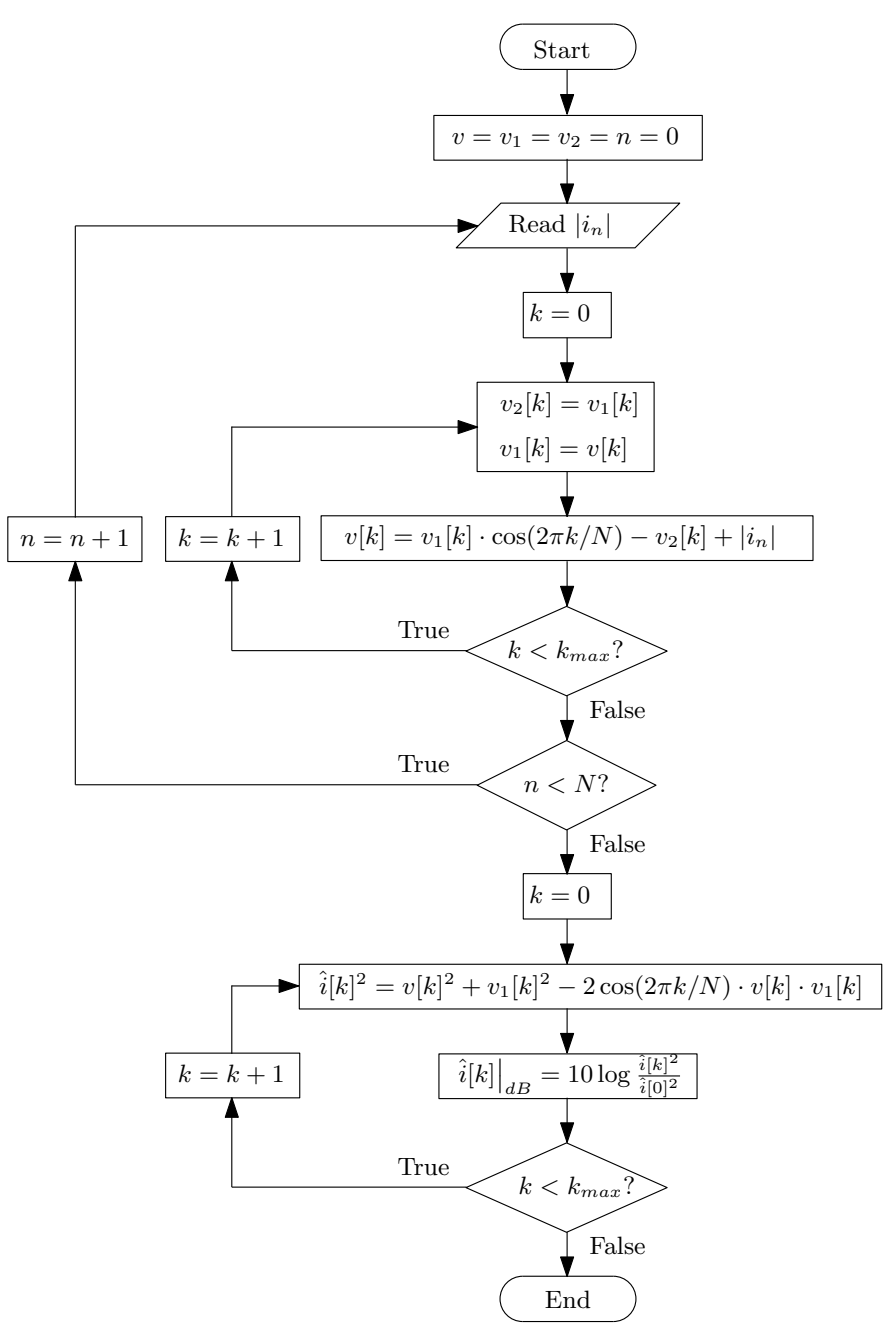

Fig. 4. Proposed implementation of the optimized Goertzel algorithm. Its recursive part is executed upon the arrival of each new rectified current sample, and is implemented using a real multiplication and two real additions per bin. The final output computes directly the modulus squared of each bin, which avoids the use of complex numbers. This algorithm is applied only at the bins with the lowest leakage generated by the supply component in the diagnostic frequency band $\left[0-2 s_{n} f_{n}\right]$, given by (17).

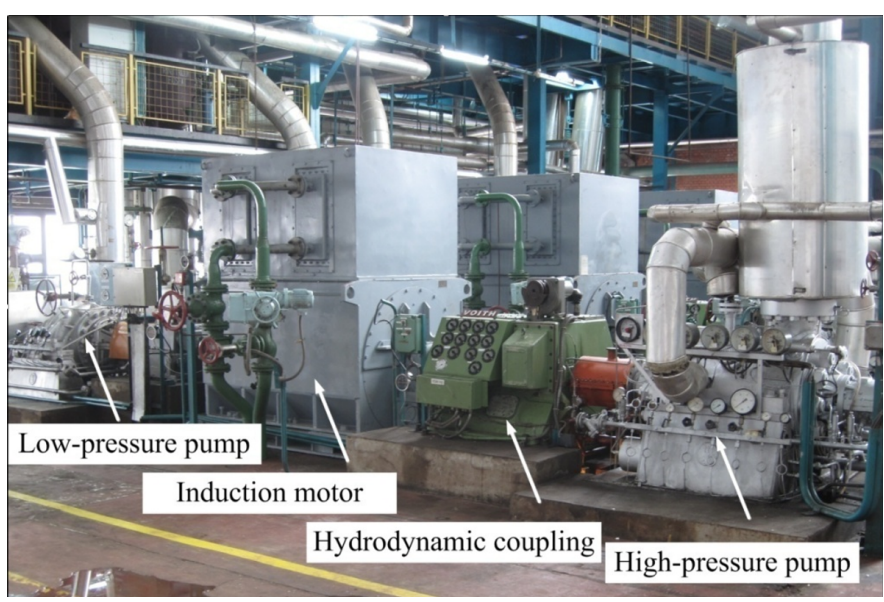

Fig. 5. IM tested with the proposed technique (see Appendix A), operating in a thermal power plant-heating plant (TPP-HP). 

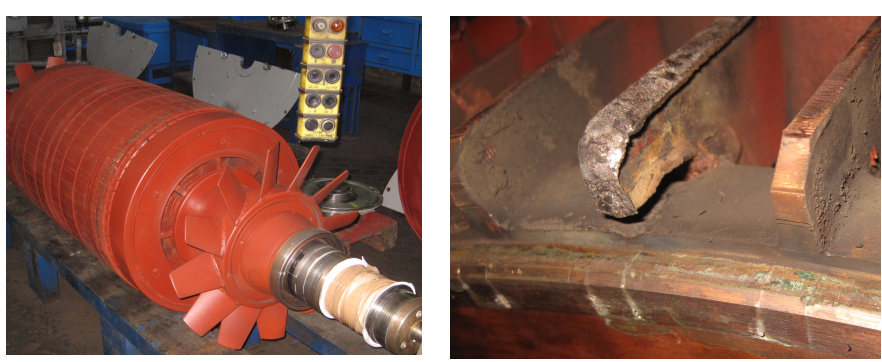

Fig. 6. Rotor of the tested 3.15 MW IM (left), and close-up view of the broken bar (right).

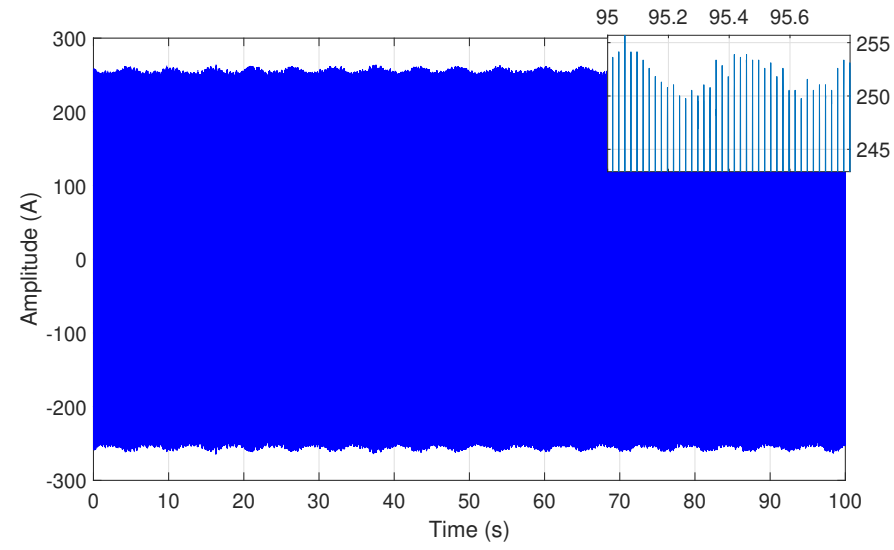

Fig. 7. Current signal obtained while the motor is partially loaded, at a constant speed of $2994.6 \mathrm{rpm}(s=0.0018)$. The enlarged view shows that, in addition to the fault components generated by the rotor asymmetry, this signal contains other components generated by electromagnetic noise, and by the non-ideal character of the machine.

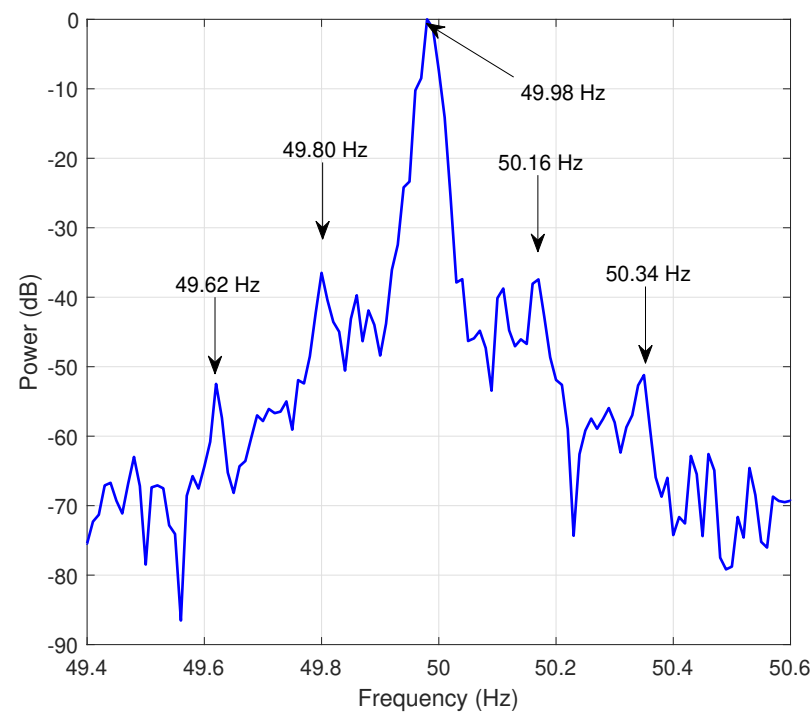

Fig. 8. Conventional spectrum of the experimental current signal of Fig. 7, using a Hanning window for reducing the spectral leakage. The broken bar harmonics calculated in Table I, second row, around the supply frequency, can be detected in this spectrum, which is an assessment of the broken bar fault, confirmed by visual inspection.
TABLE I

THEORETICAL FREQUENCIES OF THE BROKEN BAR FAULT HARMONICS IN THE TEST AT A CONSTANT SPEED OF 2994.6 RPM

\begin{tabular}{|c|c|c|c|}
\hline Fault harmonic order $k$ & 1 & 2 & 3 \\
\hline$f_{\text {asym }}=2 k s f_{1}(3)$ & $0.18 \mathrm{~Hz}$ & $0.36 \mathrm{~Hz}$ & $0.54 \mathrm{~Hz}$ \\
\hline$f_{b b}=f_{1} \pm f_{\text {asym }}(4)$ & $49.8 \mathrm{~Hz}$ & $49.62 \mathrm{~Hz}$ & $49.44 \mathrm{~Hz}$ \\
& $50.16 \mathrm{~Hz}$ & $50.34 \mathrm{~Hz}$ & $50.52 \mathrm{~Hz}$ \\
\hline
\end{tabular}

The proposed technique has been applied subsequently to obtain the spectrum of the rectified current signal of Fig. 7. This motor (see Appendix A) has a rated speed of $2982 \mathrm{rpm}$, $\left(s_{n}=0.006\right)$ and a rated frequency of $f_{n}=50 \mathrm{~Hz}$. Therefore, the algorithm of Fig. 4 has been applied to compute $k_{\max }+$ $1=61$ (16) frequency bins in the narrow spectral band $[0-$ $\left.2 s_{n} f_{n}\right]=[0-0.6] \mathrm{Hz}$, shown in Fig. 9 (hollow circles).

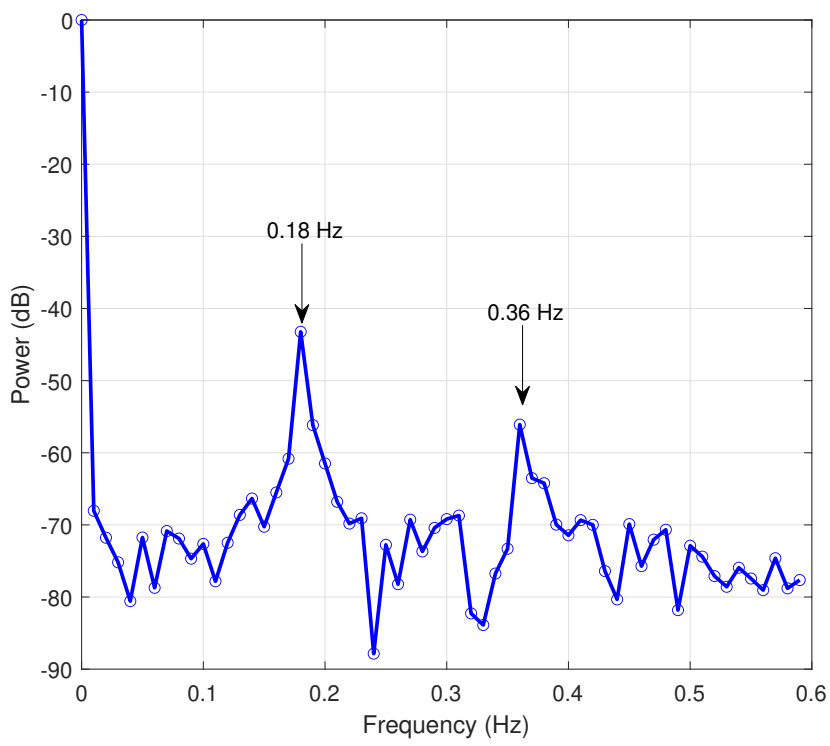

Fig. 9. Spectrum of the experimental rectified current signal of Fig. 7, generated with the optimized Goertzel algorithm (Fig. 4). The theoretical broken bar fault harmonics calculated in Table I, first row, can be detected in this spectrum, what is an indication of the broken bar fault, confirmed later by visual inspection. The circles mark the 61 points calculated by the optimized Goertzel algorithm of Fig. 4.

A direct comparison between the spectrum generated with the classical FFT approach (Fig. 8), and with the proposed approach (Fig. 9), indicates that the broken bar fault harmonics are displayed correctly in both spectra. Nevertheless, the memory needed to implement the proposed algorithm is, as indicated in Section III-C, $4\left(k_{\max }+1\right)=244$ double precision floating point numbers (1952 bytes), that is, less than $2 \mathrm{kB}$. This represents a 6000 -fold reduction compared with the classical FFT approach (11.72 MB). Besides, the peaks of the fault harmonics in Fig. 9 (without multiplying $i(t)$ by any window) are sharper than those in Fig. 8 (with a Hanning window), because the smearing effect of the window in the frequency domain is avoided. Finally, the results of the proposed approach are available right after the last current sample has been read, while the FFT approach must wait to fill the whole buffer of current samples before proceeding. As for the computing requirements, only $\left(k_{\max }+1\right)=61$ real 
multiplies and $2\left(k_{\max }+1\right)=122$ real adds are needed per current sample with the optimized Goertzel algorithm.

To further validate the proposed technique, it has been applied to the same motor in steady regime with three different loads, shown in Table II. The spectra generated by the proposed method (Fig. 4) in the tests described in Table II are depicted in Fig. 10, and show the fault harmonics at the theoretical frequencies predicted by (1). The circles in Fig. 10 mark the 61 points calculated by the algorithm of Fig. 4 in each test.

TABLE II

THEORETICAL FREQUENCIES OF THE ROTOR BROKEN BAR FAULT HARMONICS IN THREE TESTS WITH DIFFERENT LOAD LEVELS

\begin{tabular}{|c|c|c|c|c|c|}
\hline Test no. & $n$ & $s$ & $f_{1}$ & $2 s f_{1}$ & $4 s f_{1}$ \\
\hline 1 & $2996.7 \mathrm{rpm}$ & 0.0011 & $49.97 \mathrm{~Hz}$ & $0.11 \mathrm{~Hz}$ & $0.22 \mathrm{~Hz}$ \\
2 & $2995.2 \mathrm{rpm}$ & 0.0016 & $49.99 \mathrm{~Hz}$ & $0.16 \mathrm{~Hz}$ & $0.32 \mathrm{~Hz}$ \\
3 & $2994.3 \mathrm{rpm}$ & 0.0019 & $49.99 \mathrm{~Hz}$ & $0.19 \mathrm{~Hz}$ & $0.38 \mathrm{~Hz}$ \\
\hline
\end{tabular}

\section{A. Computing Times and Resources}

The proposed algorithm has been implemented on a lowcost ESP32-DEVKIT-V1 development board, whose characteristics are given in Appendix B. As the sampling frequency used in this work is $f_{s}=50 \mathrm{~Hz}$, the interval between consecutive current samples $(\Delta t=0.2 \mathrm{~ms}$ ) limits the total time available for computing the recursive part of the Goertzel algorithm of Fig. 2, the inner loop of Fig. 4. This total time per current sample, as well as the time of the non-recursive step of algorithm of Fig. 2, have been measured and are reported in Table III. Besides, as the power consumption depends on the CPU clock frequency (see Appendix B), which can be selected in this board from $80 \mathrm{MHz}$ to $240 \mathrm{MHz}$, the time measurements have been obtained for three different CPU frequencies (80, 160 and $240 \mathrm{MHz}$ ).

TABLE III

COMPUTING TIMES OF THE PROPOSED METHOD IN THE ESP32-DEVKIT-V1 DEVELOPMENT BOARD OF APPENDIX B, RUNNING AT DIFFERENT CPU CLOCK RATES.

\begin{tabular}{|c|c|c|}
\hline $\begin{array}{c}\text { CPU clock } \\
\text { rate }\end{array}$ & $\begin{array}{c}\text { Recursive part, for } \\
\text { each current sample }\end{array}$ & $\begin{array}{c}\text { Non-recursive part } \\
\text { (one execution) }\end{array}$ \\
\hline $80 \mathrm{MHz}$ & $13.8 \mu \mathrm{s}$ & $259.1 \mu \mathrm{s}$ \\
$160 \mathrm{MHz}$ & $6.8 \mu \mathrm{s}$ & $132.3 \mu \mathrm{s}$ \\
$240 \mathrm{MHz}$ & $4.8 \mu \mathrm{s}$ & $91.1 \mu \mathrm{s}$ \\
\hline
\end{tabular}

From the results presented in Table III it can be seen that, even using the lowest CPU frequency clock $(80 \mathrm{MHz})$ for energy savings purposes, the computing time per current sample of the proposed method $(13.8 \mu \mathrm{s})$ is much lower than the available time between current samples $(200 \mu \mathrm{s})$, which represents a very low computational burden, along with the minimal memory usage (1952 bytes) and code simplicity the proposed approach (Fig. 4).

\section{B. Comparison with Related Works}

The results obtained with the proposed approach are compared in this Section with other related works presented
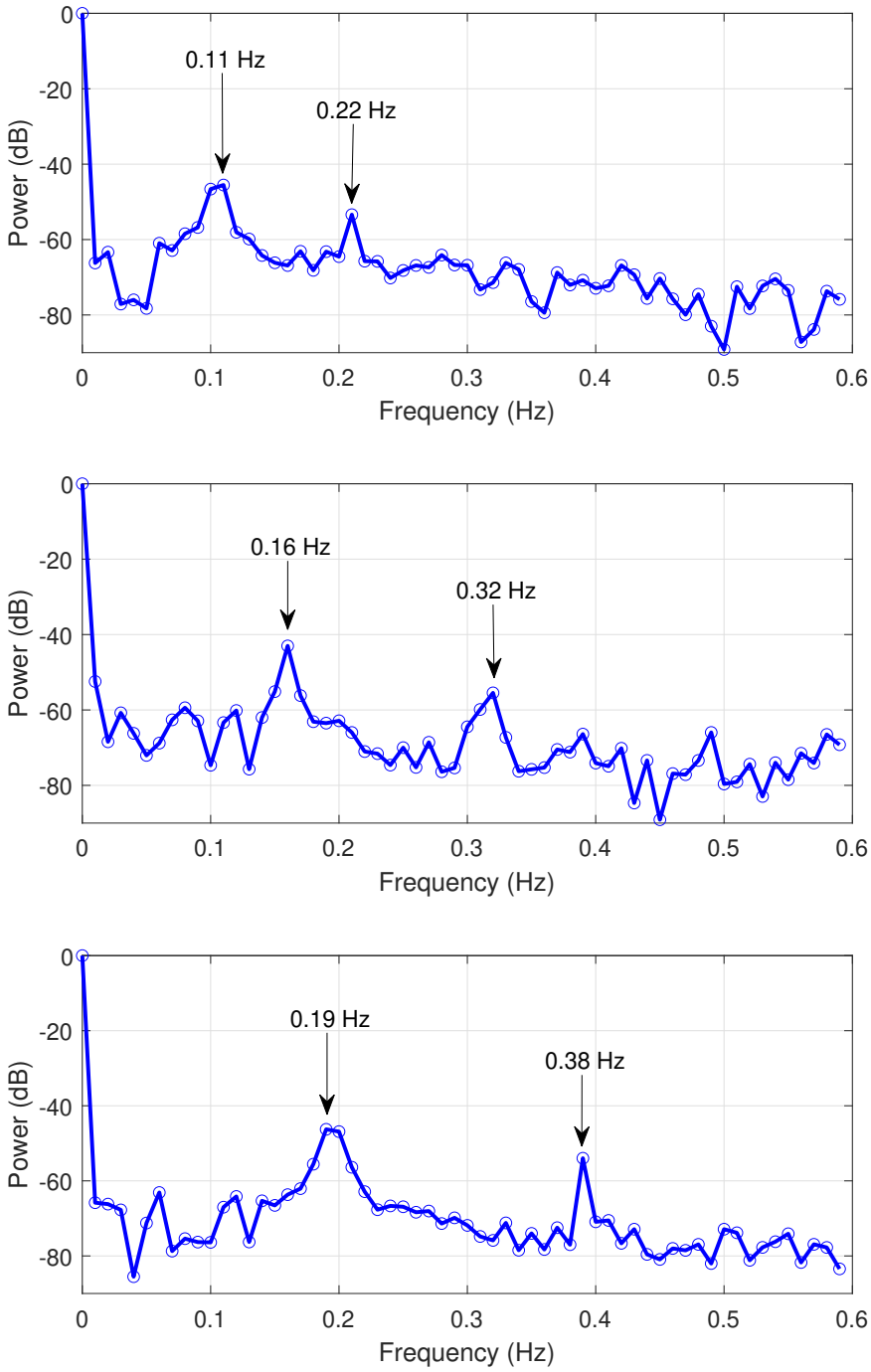

Fig. 10. Spectra of the experimental rectified current signal measured in the three tests of Table II, obtained with the proposed Goertzel algorithm of Fig. 4. Top: test $1, s=0.0011$. Middle: test $2, s=0.0016$. Bottom: test 3 , $s=0.0019$. The theoretical broken bar fault harmonics calculated in Table II coincide with the experimental fault harmonics in all the spectra, marked with arrows, what is an indication of the broken bar fault. The circles mark the 61 points calculated by the optimized Goertzel algorithm in each test.

previously in the technical literature, the Goertzel algorithm implementation proposed in [30], and the ZFFT [7], using the current of Fig. 7.

The current spectrum obtained with the Goertzel algorithm proposed in [30] is shown in Fig. 11, top. It has been applied to detect the fault harmonics in two sidebands around the fundamental frequency (121 frequency bins), which requires 484 multiplications and 242 additions for each current sample, with a computing time of $37.7 \mu$ s (with a CPU clock rate of 80 $\mathrm{MHz}$ ), and a memory storage of 3872 bytes. The output of the Goertzel algorithm is windowed with a raised cosine window to avoid the fundamental leakage. On the contrary, thanks to the use of the rectified current, the method proposed in this paper analyses only a single band starting at zero frequency (61 frequency bins), and requires only 61 multiplications and 183 additions per current sample, with a computing time of 
$13.8 \mu \mathrm{s}$ and a memory storage of 1952 bytes (a reduction of $63 \%$ in computing time and $50 \%$ in memory usage). Besides, it is not necessary to filter the output, because the proposed method is calculated directly at the bins with minimal leakage.

The current spectrum obtained with the ZFFT algorithm [7] is shown in Fig. 11, bottom. The current signal is first translated in frequency, using a digital complex mixer, and followed by a decimation stage with an anti-aliasing low-pass filter. Finally, the FFT is used for obtaining the spectrum of the decimated and windowed current signal. In this case, the final stage is a 128-point FFT, with a computing time of 461 $\mu$ s (with a CPU clock rate of $240 \mathrm{MHz}$ ). Nevertheless, using the ZFFT for detecting rotor asymmetries at very low slip has a serious drawback: a large decimation factor of 2500 has been needed to reduce the diagnostic band to a width of $2 \mathrm{~Hz}$, which requires a very narrowband computationally expensive filter. In this case, a finite impulse response (FIR) filter with 60000 elements is needed, which rises the computing time per current sample to $1007 \mu \mathrm{s}$, longer than the available time between current samples. An alternative is the use of multistage cascaded-integrator-comb filters, as in [34], which represents an increase in code complexity. Instead, in the proposed method, there is no need of frequency translation, low-pass filter, decimation, windowing process or FFT, which makes it faster and simpler to apply (only the code shown in Fig. 4 is needed).
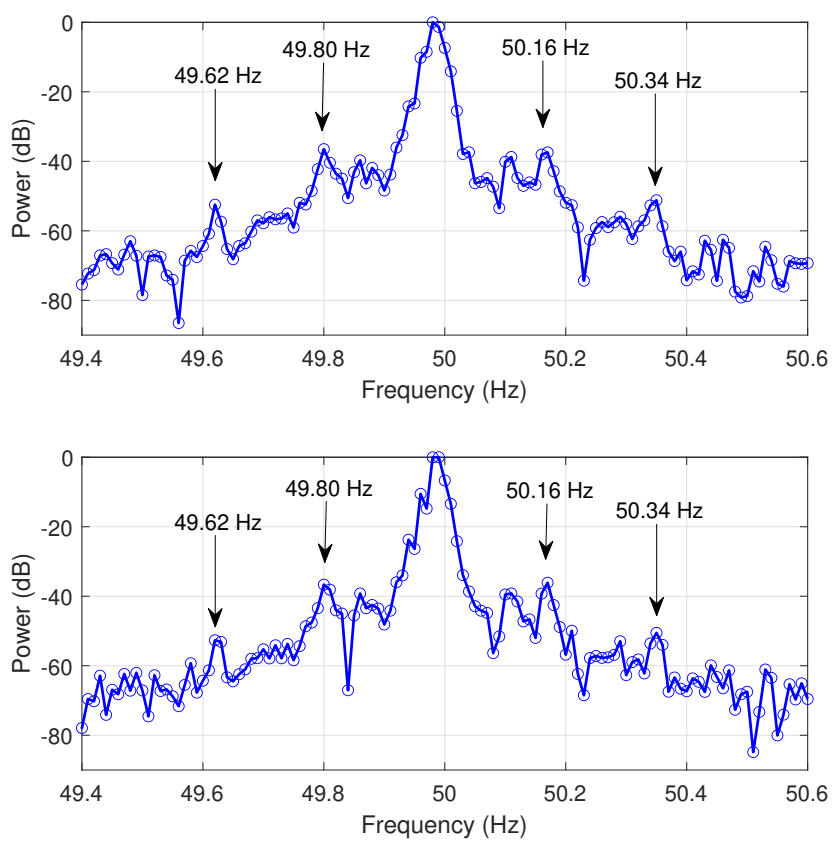

Fig. 11. Spectrum of the experimental current signal of Fig. 7, generated with the implementation of Goertzel algorithm used in [30] (top), and with the ZFFT (bottom). The circles mark the frequency bins calculated by both methods. The fault harmonics can be observed at their theoretical frequencies (marked with arrow labels), as in the case of the proposed method (see Fig. 9).

\section{Conclusions}

A novel approach has been presented in this paper to costeffectively calculate the diagnostic current spectrum of IMs operating with very low slip. The diagnosis of rotor asymmetries in these conditions is a challenging task, due to the tiny spectral distance between the dominant supply component and the fault harmonics. To obtain this high spectral resolution, long acquisition times are needed, that is, a high number of samples, what hinders the use of low-cost embedded devices. The proposed approach solves this limitation using a combination of two diagnostic procedures: the analysis of the rectified machine's current, and the implementation of an optimized Goertzel algorithm, which is executed upon the arrival of every new current sample to avoid its storage. In this way, the detection of rotor asymmetries at very low slip can be performed with a very low memory footprint and computational effort: in the case of the tested motor, just 1952 bytes of memory storage, and 61 real multiplies and 122 real adds per current sample are needed to generate the diagnostic spectrum of a current signal with 500000 samples. The extension of the proposed approach to transient conditions, and to detect other types of IM faults, is currently being developed.

\section{APPENDIX A \\ THREE-PHASE INDUCTION MOTOR}

Rated characteristics: Power $P=3150 \mathrm{~kW}$, voltage $U=6$ $\mathrm{kV}$, current $I=373 \mathrm{~A}$, supply frequency $f=50 \mathrm{~Hz}$, full-load speed $n=2982 \mathrm{rpm}$, power factor $\cos \varphi=0.92$, number of bars $=56$.

\section{APPENDIX B \\ ESP32 DEVELOPMENT BOARD}

ESP32 DEVKIT V1 Board. Processor: Tensilica Xtensa Dual-Core 32-bit LX6 microprocessor, running at 80, 160 or $240 \mathrm{MHz}$. Voltage applied to power supply pin $\mathrm{VDD}=3.3 \mathrm{~V}$. Power consumption (in modem-sleep mode): $30 \mathrm{~mA} \sim 68 \mathrm{~mA}$ (240 MHz), $27 \mathrm{~mA} \sim 44 \mathrm{~mA}(160 \mathrm{MHz}), 20 \mathrm{~mA} \sim 31 \mathrm{~mA}$ (80 MHz). ROM: 448 KB. SRAM: 520 KB. Flash memory: 4 MB. WiFi: 150.0 Mbps. Bluetooth: BLE. Peripheral Interfaces: 12-bit SAR ADC up to 18 channels, $2 \times 8$-bit DAC, $4 \times$ SPI, $2 \times \mathrm{I}^{2} \mathrm{~S}, 2 \times \mathrm{I}^{2} \mathrm{C}, 3 \times$ UART, Motor PWM, Hall sensor. Programming environment: Arduino IDE 1.8.15.c.

\section{REFERENCES}

[1] S. Karmakar, S. Chattopadhyay, M. Mitra, and S. Sengupta, "Induction Motor Fault Diagnosis: General Discussion and Research Scope," in Induction Motor Fault Diagnosis. Power Systems. Springer, Singapore, 2016, pp. 153-158.

[2] I. Culbert and J. Letal, "Signature analysis for online motor diagnostics: Early detection of rotating machine problems prior to failure," IEEE Indus. Appl. Mag., vol. 23, no. 4, pp. 76-81, 2017.

[3] M. Benbouzid, M. Vieira, and C. Theys, "Induction motors' faults detection and localization using stator current advanced signal processing techniques," IEEE Trans. Power Electron., vol. 14, no. 1, pp. 14-22, 1999.

[4] A. Naha, A. K. Samanta, A. Routray, and A. K. Deb, "Low Complexity Motor Current Signature Analysis Using Sub-Nyquist Strategy With Reduced Data Length," IEEE Trans. Instrum. Meas., vol. 66, no. 12, pp. 3249-3259, 2017.

[5] M. Benbouzid and G. Kliman, "What stator current processing-based technique to use for induction motor rotor faults diagnosis?" IEEE Trans. Energy Convers., vol. 18, no. 2, pp. 238-244, 2003.

[6] S. Nandi, H. Toliyat, and X. Li, "Condition monitoring and fault diagnosis of electrical motors-a review," IEEE Trans. Energy Convers., vol. 20 , no. 4, pp. 719-729, 2005. 
[7] A. Bellini, A. Yazidi, F. Filippetti, C. Rossi, and G.-A. Capolino, "High frequency resolution techniques for rotor fault detection of induction machines," IEEE Trans. Ind. Electron., vol. 55, no. 12, pp. 4200-4209, 2008.

[8] R. Puche-Panadero, J. Martinez-Roman, A. Sapena-Bano, J. BurrielValencia, M. Pineda-Sanchez, J. Perez-Cruz, and M. Riera-Guasp, "New Method for Spectral Leakage Reduction in the FFT of Stator Currents: Application to the Diagnosis of Bar Breakages in Cage Motors Working at Very Low Slip," IEEE Trans. Instrum. Meas., vol. 70, pp. 1-11, 2021.

[9] T. A. Garcia-Calva, D. Morinigo-Sotelo, A. Garcia-Perez, D. CamarenaMartinez, and R. de Jesus Romero-Troncoso, "Demodulation Technique for Broken Rotor Bar Detection in Inverter-Fed Induction Motor Under Non-Stationary Conditions," IEEE Trans. Energy Convers., vol. 34, no. 3, pp. 1496-1503, 2019.

[10] R. Puche-Panadero, M. Pineda-Sanchez, M. Riera-Guasp, J. RogerFolch, E. Hurtado-Perez, and J. Perez-Cruz, "Improved Resolution of the MCSA Method Via Hilbert Transform, Enabling the Diagnosis of Rotor Asymmetries at Very Low Slip," IEEE Trans. Energy Convers. vol. 24, no. 1, pp. 52-59, 2009.

[11] G. Joksimovic, J. Riger, T. Wolbank, N. Peric, and M. Vasak, "StatorCurrent Spectrum Signature of Healthy Cage Rotor Induction Machines," IEEE Trans. Ind. Electron., vol. 60, no. 9, pp. 4025-4033, 2013.

[12] M. Cabanas, F. Pedrayes, C. Rojas, M. Melero, J. Norniella, G. Orcajo, J. Cano, F. Nuno, and D. Fuentes, "A New Portable, Self-Powered, and Wireless Instrument for the Early Detection of Broken Rotor Bars in Induction Motors," IEEE Trans. Ind. Electron., vol. 58, no. 10, pp. 4917-4930, 2011.

[13] A. Lebaroud and A. Medoued, "Online computational tools dedicated to the detection of induction machine faults," International Journal of Electrical Power \& Energy Systems, vol. 44, no. 1, pp. 752-757, 2013.

[14] A. Sapena-Baño, J. Perez-Cruz, R. Puche-Panadero, J. Martinez-Roman, J. Burriel-Valencia, and J. Lazaro-Garcia, "Motor current signal analysis on programmable logic controller," in 2014 International Conference on Electrical Machines (ICEM), Berlin, Germany, Sep 2-5, 2014, pp. 16481653.

[15] C. G. Dias and F. H. Pereira, "Broken Rotor Bars Detection in Induction Motors Running at Very Low Slip Using a Hall Effect Sensor," IEEE Sensors Journal, vol. 18, no. 11, pp. 4602-4613, 2018.

[16] P. Zhang, Y. Du, T. G. Habetler, and B. Lu, "A Survey of Condition Monitoring and Protection Methods for Medium-Voltage Induction Motors," IEEE Trans. Ind Appl., vol. 47, no. 1, pp. 34-46, 2011.

[17] Y.-H. Kim, Y.-W. Youn, D.-H. Hwang, J.-H. Sun, and D.-S. Kang, "High-Resolution Parameter Estimation Method to Identify Broken Rotor Bar Faults in Induction Motors," IEEE Trans. Ind. Electron. vol. 60, no. 9, pp. 4103-4117, 2013.

[18] F. Han, L. Li, K. Wang, F. Feng, H. Pan, B. Zhang, G. He, and J. Lin, "An ultra-long FFT architecture implemented in a reconfigurable application specified processor," IEICE Electronics Express, vol. 13, no. 13, pp. $1-12,2016$.

[19] S.-Y. Lin, C.-L. Wey, and M.-D. Shieh, "Low-cost FFT processor for DVB-t2 applications," IEEE Trans. Consum. Electron., vol. 56, no. 4, pp. 2072-2079, 2010

[20] M. A. Moussa, M. Boucherma, and A. Khezzar, "A Detection Method for Induction Motor Bar Fault Using Sidelobes Leakage Phenomenon of the Sliding Discrete Fourier Transform," IEEE Trans. Power Electron., vol. 32, no. 7, pp. 5560-5572, 2017.

[21] Garcia-Perez, A. and de Jesus Romero-Troncoso, R. and Cabal-Yepez, E. and Osornio-Rios, R.A., "The Application of High-Resolution Spectral Analysis for Identifying Multiple Combined Faults in Induction Motors," IEEE Trans. Ind. Electron., vol. 58, no. 5, pp. 2002-2010, 2011.

[22] B. Xu, L. Sun, L. Xu, and G. Xu, "Improvement of the Hilbert Method via Esprit for detecting rotor fault in induction motors at low slip," IEEE Trans. Ener. Convers., vol. 28, no. 1, pp. 225-233, March 2013.

[23] A. Da Silva, N. Demerdash, and R. Povinelli, "Rotor Bar Fault Monitoring Method Based on Analysis of Air-gap Torques of Induction Motors," IEEE Trans. Ind. Informat., vol. 9, no. 4, pp. 2274-2283, 2013.

[24] J. L. Contreras-Hernandez, D. L. Almanza-Ojeda, S. Ledesma-Orozco, A. Garcia-Perez, R. J. Romero-Troncoso, and M. A. Ibarra-Manzano, "Quaternion Signal Analysis Algorithm for Induction Motor Fault Detection," IEEE Trans. Ind. Electron., vol. 66, no. 11, pp. 8843-8850, 2019.

[25] E. Jacobsen and R. Lyons, "The sliding DFT," IEEE Sig. Process. Mag., vol. 20, no. 2, pp. 74-80, Mar 2003.

[26] L. I. Gliga, B. D. Ciubotaru, H. Chafouk, D. Popescu, and C. Lupu, "Fault Diagnosis of a Direct Drive Wind Turbine Using a Bank of Goertzel Filters," in 2019 6th International Conference on Control,
Decision and Information Technologies (CoDIT), April 23-26, 2019, Paris, France, pp. 1729-1734.

[27] D. Cordoneanu and C. Nitu, "An Approach of Extracting Features for Fault Diagnosis in Bearings Using the Goertzel Algorithm," in Proceedings of the International Conference of Mechatronics and CyberMixMechatronics - 2019, Bucharest, Romania, Sept 5-6, 2019, G. I. Gheorghe, Ed. Cham: Springer International Publishing, pp. 173-183.

[28] D. V. Spyropoulos, E. D. Mitronikas, and E. S. Dermatas, "Broken Rotor Bar Fault Diagnosis in Induction Motors Using a Goertzel Algorithm," in 2018 XIII International Conference on Electrical Machines (ICEM), Alexandroupoli, Greece, Sep 3-6, 2018, pp. 1782-1788.

[29] R. Lyons, "dsp tips \& tricks - the sliding DFT," IEEE Signal Processing Magazine, vol. 20, no. 2, pp. 74-80, Mar 2003.

[30] A. Sapena-Bano, Z̆. Kanović, J. Burriel-Valencia, J. Martinez-Roman, J. Perez-Cruz, R. Puche-Panadero, M. Riera-Guasp, and M. PinedaSanchez, "Using the Goertzel Algorithm Over Disjoint Narrow Frequency Bands for Fault Diagnosis of Induction Motors," in 2018 XIII International Conference on Electrical Machines (ICEM), Alexandroupoli, Greece, Sep 3-6, 2018, pp. 1965-1971.

[31] R. Puche-Panadero, J. Martinez-Roman, A. Sapena-Bano, and J. BurrielValencia, "Diagnosis of Rotor Asymmetries Faults in Induction Machines Using the Rectified Stator Current," IEEE Trans. Energy Convers., vol. 35, no. 1, pp. 213-221, 2020.

[32] E. Jacobsen and R. Lyons, "An update to the sliding DFT," IEEE Sig. Process. Mag., vol. 21, no. 1, pp. 110-111, 2004.

[33] R. Radhouane, P. Liu, and C. Modlin, "Minimizing the memory requirement for continuous flow FFT implementation: continuous flow mixed mode FFT (CFMM-FFT)," in 2000 IEEE International Symposium on Circuits and Systems (ISCAS), May 20-31, 2000, Geneva, Switzerland, vol. 1, pp. 116-119.

[34] D. Pei, S. Yang, H. Yang, Q. Wang, and M. Li, "High efficient and realtime realization of zoom FFT based on FPGA," in 2010 International Conference on Computer Application and System Modeling (ICCASM 2010), Oct 22-24, 2010, Taiyuan, China, vol. 2, pp. 669-673. 